\title{
New polymorphic nuclear microsatellites from Aristotelia chilensis (Mol.) Stuntz (Elaeocarpaceae)
}

\author{
Matías Cona ${ }^{1}, \mathrm{M}^{\mathrm{a}}$ Herminia Castro ${ }^{1}$, Pedro León-Lobos ${ }^{1}$, Francisco Correa ${ }^{2}$, Greg Jordan ${ }^{3}$, \\ Adriana Bastías ${ }^{4}$, Boris Sagredo ${ }^{5}$, and Patricio Hinrichsen ${ }^{1 *}$ \\ ${ }^{1}$ Instituto de Investigaciones Agropecuarias, INIA La Platina, Santa Rosa 11610, Santiago, Chile. \\ *Corresponding author (phinrichsen@inia.cl). \\ ${ }^{2}$ Universidad de Talca, Facultad de Ingeniería, Av. Lircay s/n, Talca, Chile. \\ ${ }^{3}$ University of Tasmania, School of Natural Sciences, Hobart, Tas 7001, Australia. \\ ${ }^{4}$ Universidad Autónoma de Chile, Av. Pedro de Valdivia 425, Santiago, Chile. \\ ${ }^{5}$ Instituto de Investigaciones Agropecuarias, INIA Rayentué, Av. Salamanca s/n, Sector Los Choapinos, Rengo, Chile.
}

Received: 21 November 2019; Accepted: 14 January 2020; doi:10.4067/S0718-58392020000200153

\begin{abstract}
Maqui (Aristotelia chilensis [Molina] Stuntz) is a dioecious small tree native to Chile and southwestern Argentina. This species has gained attention due to its high polyphenol content and anti-oxidant capacity. Nevertheless, genetics studies and information about $A$. chilensis population genetics are scarce and even contradictory. In fact, the available species-specific simple sequence repeat (SSR) markers are not informative at all, and so we decided to identify and characterize new ones able to trace individual genotypes, a basic tool intended for different genetic studies. We identified and characterized 15 new polymorphic SSR markers for A. chilensis. These markers were evaluated in three populations distributed along 1000 $\mathrm{km}$ of Central Chile, exhibiting up to 10 alleles per locus and a combined expected heterozygosity of 0.858 . Markers were also informative in two related species, Aristotelia peduncularis (Labill.) Hook. f. and Crinodendron patagua Molina (Elaeocarpaceae), with 13 and six SSRs showing clear amplification patterns, respectively. This new set of SSR markers are highly polymorphic and informative, being the first ones available for the effective fingerprinting of maqui genotypes. A proof of concept of that was the differentiation of six maqui accessions that are under domestication for productive purposes, based on a subset of the polymorphic SSR markers.
\end{abstract}

Key words: Fingerprinting, maqui, maqui berry, molecular markers, Patagonian endemics, SSR.

\section{INTRODUCTION}

Aristotelia chilensis [Molina] Stuntz (Elaeocarpaceae), better known as maqui, is a dioecious evergreen shrub or tree (up to $6 \mathrm{~m}$ tall) endemic to Patagonia, distributed in Chile from Limarí $\left(32^{\circ} \mathrm{S}\right.$ lat) to Aysén $\left(42^{\circ} \mathrm{S}\right.$ lat) and from Coastal range to Andean slopes (2000 m a.s.1.) It is also ubiquitous in the adjacent Andean regions in Argentina. Aristotelia chilensis has been described as part of the structure of sclerophyllous heathlands and deciduous Nothofagus forests. Also, it is considered a pioneering species due to its capacity to firstly colonize disturbed soils, forming monospecific associations called "macales". Its small spherical fruits (2-5 mm diameter containing up to four seeds) are fully purple black when ripe and have been historically used as a source of food, dye and medicine mostly by Mapuche people (Misle et al., 2011). Many studies have reported high antioxidants content in its fruit, revealing maqui as one of highest antioxidant capacity among marketed fruits worldwide. Theses antioxidants include anthocyanins, tannins, flavonoids and other polyphenols: compounds that plays protective roles in oxidative stress (e.g. oxidized low-density lipoprotein [LDL]) and chronic noncommunicable diseases such as cardiovascular diseases and diabetes (Miranda-Rottmann et al., 2002; Céspedes et al., 2008; Rojo et al., 2012; Fernández et al., 2019). 
In recent years, A. chilensis has been under domestication in order to create new lines of productive interest; up to now, there are five registered varieties ('Huiña', 'Pudú', 'Vicuña', 'Puyuhuapi', and 'Taitao') in the official site of Servicio Agrícola y Ganadero (SAG) (Agriculture and Livestock Service; www.sag.gob.cl), varieties authority in Chile (Vogel et al., 2016), and new domestication initiatives are in progress. However, little is known about A. chilensis genetics although there is some information describing genetic diversity of the species based on amplified fragment length polymorphism (AFLP) (Salgado et al., 2017), inter-simple sequence repeat (ISSR) (Fredes et al., 2014) and microsatellite simple sequence repeat (SSR) (Bastías et al., 2016) markers. Nevertheless, the available SSRs were unable to genetically differentiate A. chilensis accessions from different localities, including a group of genotypes belonging to a private domestication initiative, which exhibited clear phenotypic differences. Based on the aforementioned studies, the species appears as not having clearly structured populations, and even its genetic diversity is still an open question. Considering these antecedents, and that new cultivars of vegetative propagation are expected to be released during the next years, the aim of this study was to asses an effective differentiation and traceability protocol for A. chilensis genotypes based on a new set of highly polymorphic, informative SSR markers. At the same time, these new markers could become an effective tool to re-evaluate the genetic diversity and population structure of the species.

\section{MATERIALS AND METHODS}

For the analysis of the designed primers, leaves from 60 individuals of A. chilensis were sampled in three different geographic locations in Chile (Metropolitan, Maule, and Los Lagos Regions), with 20 individuals each. These geographic locations were considered as a representative range of the species distribution. Also, diverse sampling sites were selected prioritizing wild forest populations (Table 1). Samples of the related species Crinodendron patagua Molina and Aristotelia peduncularis (Labill.) Hook. f. were obtained from sclerophyll hills close to Santiago and wet sclerophyll forests in Ferntree, Tasmania, respectively.

Sequencing and identification of new primers is described in Bastías et al. (2019). Briefly, genomic DNA was obtained from fresh leaves of maqui (Aristotelia chilensis [Molina] Stuntz). Using a library with 300 bp insert size and paired-endtag DNA sequencing using NextSeq 550 platform (Illumina, San Diego, California, USA) around 187 million $2 \times 151 \mathrm{bp}$ reads were generated. After a process of quality trimming and filtering of data using FastQC v0.11.5 (Babraham Institute, Cambridge, UK), which allow to remove reads containing more than 5\% unknown nucleotides, low-quality reads (reads containing more than $50 \%$ bases with Q-value $\leq 20)$, all unpaired reads and short reads $(<35 \mathrm{bp}), 95.87 \%$ from the total reads were suitable for genome assembling. Then, de novo assembly of the clean reads was performed to generate contigs and scaffolds. For de novo assembly, MaSuRCA 3.3.1 software (Zimin et al., 2013) was used, with optimized k-mer length of 85, calculated by KmerGenie software (Chikhi and Medvedev, 2014). The contig sequences obtained in FASTA files were screened with a repeat motif size range of 2 to $8 \mathrm{bp}$ and a length of $>12 \mathrm{bp}$. This included dinucleotide repeats $\geq 6$, trinucleotide repeats $\geq 4$, and tetra-, penta-, hexa-, hepta- and octanucleotide repeats $\geq 3$, using MIcroSAtellite identification software (Thiel et al., 2003). Then, primers were designed in the flanking regions of the 28575 found SSRs using PRIMER 3 (Rozen and Skaletsky, 2000). From this large set, we filtered each set of di, tri, tetra, penta, hepta and octanucleotide SSRs by predicted amplicon size (range of 130-280 bp) and selecting the largest motif repetitions found in each SSR type. Sixty-nine SSRs were selected (Table 2) and subjected to screening for amplification quality using a set of 10 randomly chosen A. chilensis genotypes.

Genomic DNA (gDNA) extraction was carried out using modified cetyltrimethylammonium bromide (CTAB) method with $100 \mathrm{mg}$ fresh young leaves (Lodhi et al., 1994). Yield and integrity of DNA was evaluated by electrophoresis on $1 \%$ agarose stained with ethidium bromide. Every PCR amplification contained, in a total volume of $12 \mu \mathrm{L}, 3 \mu \mathrm{L}$ gDNA (10 ng), $2.4 \mu \mathrm{L} 5 \times$ colorless GoTaq Flexi buffer (Promega, Madison, Wisconsin, USA), 1.5 $\mathrm{mM} \mathrm{MgCl} 2,0.5 \mathrm{mM}$ dNTPs $(0.125 \mathrm{mM}$ each $), 0.3 \mu \mathrm{M}$ primers, $0.5 \mathrm{U}(0.1 \mu \mathrm{L})$ of GoTaq DNA Polymerase Flexi (Promega) and completed with $\mathrm{dH}_{2} \mathrm{O}$. PCR conditions were the following: initially denaturation of 7 min at $94{ }^{\circ} \mathrm{C}$; then 40 cycles of $1 \mathrm{~min}$ at $95^{\circ} \mathrm{C}, 45 \mathrm{~s}$ at $58^{\circ} \mathrm{C}$ and $1 \mathrm{~min} 30 \mathrm{~s}$ at $72{ }^{\circ} \mathrm{C}$; finally, an incubation of 7 min at $72{ }^{\circ} \mathrm{C}$ was used for templates filling. PCR products were separated in polyacrylamide gels $(6 \%)$ and visualized using a silverstaining protocol. A known-size ladder was used to estimate allele sizes. Number of alleles and heterozygosity was calculated using GenAlEx program (Peakall and Smouse, 2012). 
Table 1. Global positioning system (GPS) for accessions of Aristotelia chilensis considered in this study.

\begin{tabular}{|c|c|c|c|c|c|}
\hline \multicolumn{2}{|c|}{ Metropolitan Region } & \multicolumn{2}{|c|}{ Maule Region } & \multicolumn{2}{|c|}{ Los Lagos Region } \\
\hline $\begin{array}{l}\text { Sample } \\
\text { number }\end{array}$ & GPS location & $\begin{array}{l}\text { Sample } \\
\text { number }\end{array}$ & GPS location & $\begin{array}{l}\text { Sample } \\
\text { number }\end{array}$ & GPS location \\
\hline \multirow[t]{2}{*}{1} & $33^{\circ} 29^{\prime} 53.9^{\prime \prime} \mathrm{S}$ & 21 & $34^{\circ} 59^{\prime} 01.5^{\prime \prime} \mathrm{S}$ & 41 & $41^{\circ} 16^{\prime} 17.3 ” \mathrm{~S}$ \\
\hline & $70^{\circ} 30^{\prime} 02.2^{\prime \prime} \mathrm{W}$ & & $71^{\circ} 13^{\prime} 58.2^{\prime \prime} \mathrm{W}$ & & $72^{\circ} 49^{\prime} 31.2^{\prime \prime} \mathrm{W}$ \\
\hline \multirow[t]{2}{*}{2} & $33^{\circ} 29^{\prime} 53.3^{\prime \prime} \mathrm{S}$ & 22 & $34^{\circ} 59^{\prime} 01.6^{\prime \prime} \mathrm{S}$ & 42 & $41^{\circ} 15^{\prime} 50.6 ” \mathrm{~S}$ \\
\hline & $70^{\circ} 30^{\prime} 00.8^{\prime \prime} \mathrm{W}$ & & $71^{\circ} 13^{\prime} 55.8^{\prime \prime} \mathrm{W}$ & & $72^{\circ} 48^{\prime} 55.0^{\prime \prime} \mathrm{W}$ \\
\hline \multirow[t]{2}{*}{3} & $33^{\circ} 29^{\prime} 51.7^{\prime \prime} \mathrm{S}$ & 23 & $34^{\circ} 58^{\prime} 53.5^{\prime \prime} \mathrm{S}$ & 43 & $41^{\circ} 11^{\prime} 44.9 " \mathrm{~S}$ \\
\hline & $70^{\circ} 29^{\prime} 59.5 ” \mathrm{~W}$ & & $71^{\circ} 13^{\prime} 46.4^{\prime \prime} \mathrm{W}$ & & $72^{\circ} 32^{\prime} 22.9^{\prime \prime} \mathrm{W}$ \\
\hline \multirow[t]{2}{*}{4} & $33^{\circ} 29^{\prime} 51.5^{\prime \prime} \mathrm{S}$ & 24 & $34^{\circ} 58^{\prime} 53.2^{\prime \prime} \mathrm{S}$ & 44 & $41^{\circ} 11^{\prime} 44.77^{\prime \prime S}$ \\
\hline & $70^{\circ} 29^{\prime} 53.5^{\prime \prime} \mathrm{W}$ & & $71^{\circ} 13^{\prime} 47.6^{\prime \prime} \mathrm{W}$ & & $72^{\circ} 32^{\prime} 23.1^{\prime \prime} \mathrm{W}$ \\
\hline \multirow[t]{2}{*}{5} & $33^{\circ} 29^{\prime} 52.4^{\prime \prime} \mathrm{S}$ & 25 & $34^{\circ} 52^{\prime} 40.8^{\prime \prime} \mathrm{S}$ & 45 & $41^{\circ} 07^{\prime} 33.0^{\prime \prime} \mathrm{S}$ \\
\hline & $70^{\circ} 29^{\prime} 51.1 ” \mathrm{~W}$ & & $71^{\circ} 20^{\prime} 42.7^{\prime \prime} \mathrm{W}$ & & $72^{\circ} 36^{\prime} 47.1^{\prime \prime} \mathrm{W}$ \\
\hline \multirow[t]{2}{*}{6} & $33^{\circ} 29^{\prime} 54.5^{\prime \prime} \mathrm{S}$ & 26 & $34^{\circ} 52^{\prime} 27.7^{\prime \prime} \mathrm{S}$ & 46 & $41^{\circ} 07^{\prime} 28.0 ” \mathrm{~S}$ \\
\hline & $70^{\circ} 29^{\prime} 46.8^{\prime \prime} \mathrm{W}$ & & $71^{\circ} 20^{\prime} 52.3^{\prime \prime} \mathrm{W}$ & & $72^{\circ} 36^{\prime} 52.2^{\prime \prime} \mathrm{W}$ \\
\hline \multirow[t]{2}{*}{7} & $33^{\circ} 29^{\prime} 55.8^{\prime \prime} \mathrm{S}$ & 27 & $34^{\circ} 52^{\prime} 27.1 ” \mathrm{~S}$ & 47 & $41^{\circ} 04^{\prime} 25.1^{\prime \prime} \mathrm{S}$ \\
\hline & $70^{\circ} 29^{\prime} 10.8^{\prime \prime} \mathrm{W}$ & & $71^{\circ} 20^{\prime} 53.5^{\prime \prime} \mathrm{W}$ & & $72^{\circ} 36^{\prime} 59.1 ” \mathrm{~W}$ \\
\hline \multirow[t]{2}{*}{8} & $33^{\circ} 29^{\prime} 52.1 " \mathrm{~S}$ & 28 & $34^{\circ} 51^{\prime} 22.7^{\prime \prime} \mathrm{S}$ & 48 & $41^{\circ} 04^{\prime} 31.8^{\prime \prime} \mathrm{S}$ \\
\hline & $70^{\circ} 29^{\prime} 05.1 ” \mathrm{~W}$ & & $71^{\circ} 21^{\prime} 11.2 ” \mathrm{~W}$ & & $72^{\circ} 37^{\prime} 15.9^{\prime \prime} \mathrm{W}$ \\
\hline \multirow[t]{2}{*}{9} & $33^{\circ} 29^{\prime} 48.2^{\prime \prime} \mathrm{S}$ & 29 & $34^{\circ} 51^{\prime} 12.6 ” \mathrm{~S}$ & 49 & $40^{\circ} 59^{\prime} 46.8^{\prime \prime} \mathrm{S}$ \\
\hline & $70^{\circ} 29^{\prime} 01.8^{\prime \prime} \mathrm{W}$ & & $71^{\circ} 21^{\prime} 08.9^{\prime \prime} \mathrm{W}$ & & $72^{\circ} 45^{\prime} 33.3^{\prime \prime} \mathrm{W}$ \\
\hline \multirow[t]{2}{*}{10} & $33^{\circ} 29^{\prime} 45.3^{\prime \prime} \mathrm{S}$ & 30 & $34^{\circ} 50 ’ 21.1 ” \mathrm{~S}$ & 50 & $41^{\circ} 40^{\prime} 14.6 ” \mathrm{~S}$ \\
\hline & $70^{\circ} 28^{\prime} 57.0^{\prime \prime} \mathrm{W}$ & & $71^{\circ} 21^{\prime} 10.9 ” \mathrm{~W}$ & & $73^{\circ} 03^{\prime} 58.9^{\prime \prime} \mathrm{W}$ \\
\hline \multirow[t]{2}{*}{11} & $33^{\circ} 29^{\prime} 43.3^{\prime \prime} \mathrm{S}$ & 31 & $34^{\circ} 50^{\prime} 21.4^{\prime \prime} \mathrm{S}$ & 51 & $41^{\circ} 40^{\prime} 13.1^{\prime \prime} \mathrm{S}$ \\
\hline & $70^{\circ} 28^{\prime} 51.1 ” \mathrm{~W}$ & & $71^{\circ} 21^{\prime} 12.8^{\prime \prime} \mathrm{W}$ & & $73^{\circ} 03^{\prime} 59.8^{\prime \prime} \mathrm{W}$ \\
\hline \multirow[t]{2}{*}{12} & $33^{\circ} 29^{\prime} 42.7^{\prime} \mathrm{S}$ & 32 & $34^{\circ} 50^{\prime} 19.7 ” \mathrm{~S}$ & 52 & $41^{\circ} 40^{\prime} 12.4 " \mathrm{~S}$ \\
\hline & $70^{\circ} 28^{\prime} 45.6^{\prime \prime} \mathrm{W}$ & & $71^{\circ} 21^{\prime} 12.9^{\prime \prime} \mathrm{W}$ & & $73^{\circ} 04^{\prime} 04.4^{\prime \prime} \mathrm{W}$ \\
\hline \multirow[t]{2}{*}{13} & $33^{\circ} 29^{\prime} 38.1^{\prime \prime} \mathrm{S}$ & 33 & $34^{\circ} 50^{\prime} 19.3 " \mathrm{~S}$ & 53 & $41^{\circ} 40^{\prime} 12.8^{\prime \prime} \mathrm{S}$ \\
\hline & $70^{\circ} 28^{\prime} 40.1 ” \mathrm{~W}$ & & $71^{\circ} 21^{\prime} 13.8^{\prime \prime} \mathrm{W}$ & & $73^{\circ} 03^{\prime} 57.7^{\prime \prime} \mathrm{W}$ \\
\hline \multirow[t]{2}{*}{14} & $33^{\circ} 29^{\prime} 35.5^{\prime \prime} \mathrm{S}$ & 34 & $34^{\circ} 50^{\prime} 19.6 ” \mathrm{~S}$ & 54 & $41^{\circ} 40^{\prime} 08.0^{\prime \prime} \mathrm{S}$ \\
\hline & $70^{\circ} 28^{\prime} 34.8^{\prime \prime} \mathrm{W}$ & & $71^{\circ} 21^{\prime} 15.0^{\prime \prime} \mathrm{W}$ & & $73^{\circ} 03^{\prime} 50.9^{\prime \prime} \mathrm{W}$ \\
\hline \multirow[t]{2}{*}{15} & $33^{\circ} 29^{\prime} 36.4^{\prime \prime} \mathrm{S}$ & 35 & $34^{\circ} 50^{\prime} 19.1 ” \mathrm{~S}$ & 55 & $41^{\circ} 40^{\prime} 09.6 ” \mathrm{~S}$ \\
\hline & $70^{\circ} 31^{\prime} 05.0{ }^{\prime \prime} \mathrm{W}$ & & $71^{\circ} 21^{\prime} 15.3 ” \mathrm{~W}$ & & $73^{\circ} 03^{\prime} 50.7^{\prime \prime} \mathrm{W}$ \\
\hline \multirow[t]{2}{*}{16} & $33^{\circ} 29^{\prime} 49.3^{\prime \prime} \mathrm{S}$ & 36 & $34^{\circ} 50^{\prime} 18.4^{\prime \prime} \mathrm{S}$ & 56 & $41^{\circ} 29^{\prime} 11.2 " \mathrm{~S}$ \\
\hline & $70^{\circ} 54^{\prime} 31.0 " \mathrm{~W}$ & & $71^{\circ} 21^{\prime} 15.6$ " W & & $72^{\circ} 56^{\prime} 57.4$ " W \\
\hline \multirow[t]{2}{*}{17} & $33^{\circ} 29^{\prime} 47.4^{\prime \prime} \mathrm{S}$ & 37 & $34^{\circ} 50^{\prime} 18.5^{\prime \prime} \mathrm{S}$ & 57 & $41^{\circ} 29^{\prime} 11.4 " \mathrm{~S}$ \\
\hline & $70^{\circ} 54^{\prime} 34.2^{\prime \prime} \mathrm{W}$ & & $71^{\circ} 21^{\prime} 17.3 ” \mathrm{~W}$ & & $72^{\circ} 56^{\prime} 57.8^{\prime \prime} \mathrm{W}$ \\
\hline \multirow[t]{2}{*}{18} & $33^{\circ} 29^{\prime} 50.4^{\prime \prime} \mathrm{S}$ & 38 & $34^{\circ} 50^{\prime} 13.3 ” \mathrm{~S}$ & 58 & $41^{\circ} 29^{\prime} 12.2^{\prime \prime} \mathrm{S}$ \\
\hline & $70^{\circ} 54^{\prime} 41.0^{\prime \prime} \mathrm{W}$ & & $71^{\circ} 21^{\prime} 13.1^{\prime \prime} \mathrm{W}$ & & $72^{\circ} 57^{\prime} 00.6^{\prime \prime} \mathrm{W}$ \\
\hline \multirow[t]{2}{*}{19} & $33^{\circ} 29^{\prime} 50.8^{\prime \prime} \mathrm{S}$ & 39 & $34^{\circ} 50^{\prime} 11.3 " \mathrm{~S}$ & 59 & $41^{\circ} 29^{\prime} 08.0^{\prime \prime} \mathrm{S}$ \\
\hline & $70^{\circ} 54^{\prime} 45.7 ” \mathrm{~W}$ & & $71^{\circ} 21^{\prime} 17.1^{\prime \prime} \mathrm{W}$ & & $72^{\circ} 57^{\prime} 14.7$ ' W \\
\hline \multirow[t]{2}{*}{20} & $33^{\circ} 29^{\prime} 52.2^{\prime \prime} \mathrm{S}$ & 40 & $34^{\circ} 50^{\prime} 09.0^{\prime \prime} \mathrm{S}$ & 60 & $41^{\circ} 29^{\prime} 14.4 ” \mathrm{~S}$ \\
\hline & $70^{\circ} 54^{\prime} 46.7^{\prime \prime} \mathrm{W}$ & & $71^{\circ} 21^{\prime} 20.0^{\prime \prime} \mathrm{W}$ & & $72^{\circ} 57^{\prime} 25.7^{\prime \prime} \mathrm{W}$ \\
\hline
\end{tabular}

\section{RESULTS AND DISCUSSION}

Of the 69 SSRs initially screened (Table 3) 15 were dinucleotide, 13 trinucleotide, 23 tetranucleotide, four pentanucleotide, nine hexanucleotide and five heptanucleotide; eight of these SSRs showed monomorphic profiles. From the 61 remaining SSRs, 46 showed no amplification or complex patterns and 15 exhibited highly polymorphic profiles as well as clear and strong PCR signals (Table 2). These 15 SSRs were analyzed in three populations of A. chilensis $(\mathrm{n}=60)$. After this, number of alleles, as well as expected and observed heterozygosity were determined for each population (Table 4). Alleles number ranged from 1 to 10 and expected heterozygosity varied from 0 to 0.858 per locus. It was interesting to note that the monomorphic profiles in two loci (Ach45 and Ach54) were evidenced in the southernmost population sampled in this study (Los Lagos Region). Based on the last glacial maximum (LGM) Patagonian ice sheet range (Moreno et al., 2018), we hypothesize that colonization event of Los Lagos population took place more recently than those populations northward, leading to a loss of alleles and decreased genetic diversity southward this region, explained by genetic drift 
considering bottleneck or founder effects (Hewitt, 2004; Gugerli et al., 2009; Tóth et al., 2019). However, this genetic pattern remains to be studied, and new northern- and southernmost populations of A. chilensis should be considered in order to define possible refugia of this species during LGM period.

Table 2. General characteristics of 15 polymorphic SSRs markers for Aristotelia chilensis.

\begin{tabular}{|c|c|c|c|c|}
\hline Locus & Primer sequences $\left(5^{\prime}-3^{\prime}\right)$ & Repeat motif & Size range (bp) & $T_{\mathrm{a}}\left({ }^{\circ} \mathrm{C}\right)$ \\
\hline \multirow[t]{2}{*}{ Ach3 } & F: CTGTCCTCAGCTGGTATTTA & $(\mathrm{AG})_{27}$ & $252-266$ & 58 \\
\hline & R: CCTTCTTTGGTTTCTTGCTTTA & & & \\
\hline \multirow[t]{2}{*}{ Ach4 } & F: TGCACATGAAACACAGAATTA & $(\mathrm{AG})_{28}$ & $238-270$ & 58 \\
\hline & R: GCAACATGCGAGTGAAATTA & & & \\
\hline \multirow[t]{2}{*}{ Ach5 } & F: TGATGGGTTTTTGTGCAAATT & $(\mathrm{AG})_{68}$ & $194-214$ & 58 \\
\hline & R: TGATCCTGCATTTCTTTCAATAG & & & \\
\hline \multirow[t]{2}{*}{ Ach11 } & F: AGGATGCCAACCTTTTCTAAT & $(\mathrm{GA})_{22}$ & $248-276$ & 58 \\
\hline & R: CCTTTCTCTGCATTCTCTTTTT & & & \\
\hline \multirow[t]{2}{*}{ Ach14 } & F: ACTTGTTACAAACCAAACAATTT & $(\mathrm{GA})_{25}$ & $234-264$ & 58 \\
\hline & R: TGCTTCCCATTTTCTTCTTTTT & & & \\
\hline \multirow[t]{2}{*}{ Ach28 } & F: TGCGGAGAAGTAAGTGTATT & $(\mathrm{TTG})_{10}$ & $200-230$ & 58 \\
\hline & R: TGAACCACAGCTGAATGATAT & & & \\
\hline \multirow[t]{2}{*}{ Ach29 } & F: TGGAATCATGGCCTCTACTA & $(\mathrm{AAAG})_{6}$ & $200-230$ & 58 \\
\hline & R: AATCTTCCCCAACGGTATTC & & & \\
\hline \multirow[t]{2}{*}{ Ach38 } & F: TTCCTTCTCGGCTTTCTTAA & $(\mathrm{ATCT})_{7}$ & $170-194$ & 58 \\
\hline & R: GCAAACCCTAGTTCCAATTT & & & \\
\hline \multirow[t]{2}{*}{ Ach41 } & F: ACTCACTGTGGAGATTTCAATA & $(\mathrm{GAGT})_{7}$ & $196-218$ & 58 \\
\hline & R: GCCTTTTGCCAGACTTAGTA & & & \\
\hline \multirow[t]{2}{*}{ Ach 45} & F: GGAGCCGATCACGTAGTA & $(\mathrm{TACA})_{8}$ & $190-202$ & 58 \\
\hline & R: GCTGATTCGATGGGGATAAT & & & \\
\hline \multirow[t]{2}{*}{ Ach47 } & F: AGTGGCTCAGGTTTGTAATT & $(\text { TGTA })_{8}$ & $156-164$ & 58 \\
\hline & R: TGGTGGATTCCAGATTGTTAT & & & \\
\hline \multirow[t]{2}{*}{ Ach54 } & F: CTGAGAAGCACCCAAAGATA & $\left(\right.$ TAAAA $_{6}$ & $222-230$ & 58 \\
\hline & R: GGGCCAATCAGAGCTAATC & & & \\
\hline \multirow[t]{2}{*}{ Ach57 } & F: CTCCCACAACAAGACCATAA & $(\mathrm{ACCAGA})^{5}$ & $258-270$ & 58 \\
\hline & R: AACTCCGAGTTGAGAAAATT & & & \\
\hline \multirow[t]{2}{*}{ Ach61 } & F: CCTATTGCACTCACCTGATAT & $(\mathrm{GAAGTT})_{6}$ & $212-230$ & 58 \\
\hline & R: AGAGCTGGACAGACTGAATA & & & \\
\hline \multirow[t]{2}{*}{ Ach68 } & F: GGTTGAGTGGGTTGACTATT & $(\text { TATCATC })_{5}$ & $184-206$ & 58 \\
\hline & R: AGAGCAAATCAAACCAACATTA & & & \\
\hline
\end{tabular}

$T_{\mathrm{a}}$ : Annealing temperature.

Table 3. List of 69 dinucleotidic to heptanucleotidic microsatellite repeats (SSRs) from Aristotelia chilensis considered in this work.

\begin{tabular}{|c|c|c|c|c|}
\hline SSR ID & Scaffold number & Forward primer $\left(5^{\prime}-3^{\prime}\right)$ & Reverse primer $\left(5^{\prime}-3^{\prime}\right)$ & SSR motif \\
\hline Ach1 & 27262 & CATCTTGTCGCATGTGTATAC & ACATCCAAAAGCCCCATTAA & $(\mathrm{AG}) 22$ \\
\hline Ach2 & 12158 & TGGTTGTGGAGGAATGAATTA & GGATGGAGATGGTATGGTTAAT & (AG) 25 \\
\hline Ach3 & 7421 & CTGTCCTCAGCTGGTATTTA & СCTTCTTTGGTTTCTTGCTTTA & (AG) 27 \\
\hline Ach4 & 672 & TGCACATGAAACACAGAATTA & GCAACATGCGAGTGAAATTA & $(\mathrm{AG}) 28$ \\
\hline Ach5 & 38123 & TGATGGGTTTTTGTGCAAATT & TGATCCTGCATTTCTTTCAATAG & $(\mathrm{AG}) 68$ \\
\hline Ach6 & 677 & CAGCCGTCAGTTTGCTATAT & TCTCTTCACGCAACCATTAA & (CT) 21 \\
\hline Ach7 & 678 & GTGGAAGTGGTGGTCATAAT & AGCTTCCAAAGACAAGTTAGTA & (CT) 22 \\
\hline Ach8 & 22137 & TGGAAACTTCAACAACACTAAA & GCTACAGGGAGAGAGATAGA & $(\mathrm{GA}) 20$ \\
\hline Ach9 & 1006 & AACTCAGCAGACACTGTTAT & GAAATCCTTGCACCCAAAAT & $(\mathrm{GA}) 21$ \\
\hline Ach10 & 32056 & ACAGTCCCAAATGGCATTTT & САTТCСАТТССТССАСАТTTT & $(\mathrm{GA}) 22$ \\
\hline Ach11 & 1079 & AGGATGCCAACCTTTTCTAAT & ССТTTCTCTGCATTCTCTTTTT & $(\mathrm{GA}) 22$ \\
\hline Ach12 & 23751 & СТСАСТСТTGCTCСТCATTA & CATCCTTGGCCTTTGAAATT & $(\mathrm{GA}) 24$ \\
\hline Ach13 & 7717 & TCAGCATGGTCTAAGGAAAAA & AACGAGCTTTGTCACTATTG & (GA) 25 \\
\hline Ach14 & 25455 & ACTTGTTACAAACCAAACAATTT & TGCTTCCCATTTTCTTCTTTTT & (GA) 25 \\
\hline Ach15 & 9396 & TGTGCTACAACTGTGCTATT & GTGGCTATGCAGAACCTAAT & $(\mathrm{GA}) 28$ \\
\hline Ach16 & 2152 & CTGATGCCGCGTTTAATATC & CAATTGCTTCACCGCTAATC & $(\mathrm{AGT}) 14$ \\
\hline Ach17 & 40659 & GGAGGGAACAAATTCATCAAAAT & GATGTTGATAGCGAGGATTTT & (ATC) 10 \\
\hline
\end{tabular}


Continuation Table 3.

\begin{tabular}{|c|c|c|c|c|}
\hline SSR ID & Scaffold number & Forward primer (5'-3') & Reverse primer (5'-3') & SSR motif \\
\hline Ach18 & 7124 & TCACAAAAGGATGCCCATAT & TTTCTGCCACAGGGATAGAT & (ATG) 11 \\
\hline Ach19 & 12255 & ACACCCAAACAACACAAAAA & GTGGTGATGAAGGCAATAAG & (CAA) 10 \\
\hline $\operatorname{Ach} 20$ & 19103 & GTTGCCATGATCCCTCTAAT & GCTATCTGCCCAAAGGTAAT & $(\mathrm{CTT}) 10$ \\
\hline $\operatorname{Ach} 21$ & 3676 & GCCACСТСТTCTTCCATAAA & GTGGAAGAGAAGAGCTAGTA & (CTT) 12 \\
\hline $\operatorname{Ach} 22$ & 56 & CTGGATCTTGAGGAGCTTAA & CAAGTGCAACCCCTTTTTAG & (GTA) 11 \\
\hline Ach23 & 36471 & AAGCACTGGTTAGGCTTTAA & GCAAACAAACACCACAAAAA & (TCT) 10 \\
\hline Ach24 & 3236 & ATTGCCTCAAAACCCATCTA & GGGTGCAAAGATGGTGATAT & (TCT) 11 \\
\hline Ach25 & 9487 & TCAACTTTCTCAGATGCTTTTT & AGAAACATTGCCCCTCAATA & (TGA) 10 \\
\hline Ach26 & 5437 & TGGGTTTGCTACTTGTCATAT & CACCCATTCACCATGTAAAA & (TGA) 13 \\
\hline $\operatorname{Ach} 27$ & 18876 & GGAGACTTGAGAGGGTTTTT & CTGACCCCACATCTTCTAAA & (TGT) 10 \\
\hline $\operatorname{Ach} 28$ & 578 & TGCGGAGAAGTAAGTGTATT & TGAACCACAGCTGAATGATAT & (TTG) 10 \\
\hline Ach29 & 32305 & TGGAATCATGGCCTCTACTA & AATCTTCCCCAACGGTATTC & (AAAG)6 \\
\hline $\operatorname{Ach} 30$ & 18127 & ACATGCGCATTTGATTGATAA & TGTGGGCGGTACTAATACTA & (AACT)6 \\
\hline Ach31 & 2911 & TGTAGCAAACGGTTGGATTA & СТCTTGACCAAAGCTCTTTTT & $(\mathrm{AAGA}) 6$ \\
\hline Ach32 & 24448 & TGCCGTCAATGAAAGAAAAA & TGAAGGGAATTGGGGTTTTT & (ACAA) 6 \\
\hline Ach33 & 6006 & CCGCCCACGCTATTTTAA & TTGGCTTGCTTGAGGTTAAT & (AGTT)6 \\
\hline Ach34 & 1795 & TGTTTTTGCCTCAGCTTAAG & GTGCACCAAACTAGCATTTT & (ATAA) 6 \\
\hline Ach35 & 36883 & ACTTCCTACGAATCGCATTA & TTGTATGTGGGTCTCAATTT & (ATAA) 6 \\
\hline Ach36 & 2369 & AACGTGTAAGCTACCATCTA & CGGTACCGCTTTTACTAAAA & $($ ATAC) 6 \\
\hline Ach37 & 19251 & GGTGGTTAAGCTGTCAATCTA & GGAGACAAGAAAAGGCAAAAT & (ATCA)7 \\
\hline $\operatorname{Ach} 38$ & 17612 & TTCCTTCTCGGCTTTCTTAA & GCAAACCCTAGTTCCAATTT & (ATCT) 7 \\
\hline Ach39 & 13652 & TAATTCTGGCCGCAACTTAA & TGGTCGAAACCCTGAAAAAT & (CATA) 6 \\
\hline Ach40 & 19425 & GGAAGGCACACATCAGTTAT & CTGTAGCCTCATGCATCTAT & (CATA) 9 \\
\hline Ach41 & 12025 & ACTCACTGTGGAGATTTCAATA & GCCTTTTGCCAGACTTAGTA & $(\mathrm{GAGT}) 7$ \\
\hline Ach42 & 1563 & CCATGGCCACATATGTAGAT & TGTCATGCCCACCTTTAAAA & (GTAA) 8 \\
\hline Ach43 & 31317 & ATTCTCTTCTGCGCCATTAT & GGCAGATCGTCGTCAATATA & (GTCT)6 \\
\hline Ach44 & 12669 & AAGAATGGGAGGCAGTATTC & ACGCTACTCGAATCGTAATT & (GTTA)6 \\
\hline Ach45 & 20543 & GGAGCCGATCACGTAGTA & GCTGATTCGATGGGGATAAT & (TACA) 8 \\
\hline Ach46 & 2678 & CCCAGTTCCCCTTCAAATAA & AAAGTGCCACTTGACAAAAA & (TATC) 7 \\
\hline Ach47 & 17109 & AGTGGCTCAGGTTTGTAATT & TGGTGGATTCCAGATTGTTAT & (TGTA) 8 \\
\hline Ach48 & 10951 & CACGTGAAGCGAATACATAA & CAAAAACTGGACCCGTTTTA & (TTAG) 7 \\
\hline Ach49 & 40246 & CCATACCACGCCTCTTTTAT & GTTGGGTCTGGGATACTAAA & (TTAT) 7 \\
\hline Ach50 & 9790 & ACCCCAAATTGTTGCAAAAA & CTGTCCATGCTAAGATCAATTT & (TTTC) 6 \\
\hline Ach51 & 24781 & CССТTTTTACAGGCACAATTT & CAATGAACTTGCGCACATAT & (TTTC) 6 \\
\hline Ach52 & 12162 & TTGTGTGCACGTACCATTAT & GCATTTGCTAAACGTGTAGAT & $(\mathrm{AAAAG}) 6$ \\
\hline Ach53 & 36032 & TCTGAAATGAGGAAAGCATTTT & ACCTGTAGCCCTAGTCATAT & (AAAAG)6 \\
\hline Ach54 & 15539 & CTGAGAAGCACCCAAAGATA & GGGCCAATCAGAGCTAATC & (TAAAA) 6 \\
\hline Ach55 & 15669 & CTTGGGTGGGGGAATAAAG & CTGCCTAGGACTTGACATAA & $(\mathrm{TCAAC}) 5$ \\
\hline Ach56 & 38303 & GCAGTTTGAACGTGGTAATC & AAAGAGTTGGGGGTGATATG & (AAAAAG) 5 \\
\hline Ach57 & 2031 & CTCССАСАACAAGACCATAA & AACTCCGAGTTGAGAAAATT & $(\mathrm{ACCAGA}) 5$ \\
\hline Ach58 & 710 & ACGCATGACCCAAACTAAAT & GAACACACAACAACAGTTTTT & $($ ATATAC) 5 \\
\hline Ach59 & 8794 & TGTTCCCATCTCATGCTAAT & CTGACTTGTGCCTCCTTTTA & $(\mathrm{CAATCA}) 5$ \\
\hline Ach60 & 13927 & TGCAGTGAAACAAGTGATTTT & GTGAAAAGGTTAGGTAGCTAAA & $($ CTAAAT) 6 \\
\hline Ach61 & 14205 & CCTATTGCACTCACCTGATAT & AGAGCTGGACAGACTGAATA & (GAAGTT)6 \\
\hline Ach62 & 874 & CTGGCAGGGAGAGTTAAAAA & TGGCACAAAACTGGATAATG & (TTGGTA) 6 \\
\hline Ach63 & 7478 & TCCACAACCAAAGATTCTTATG & TGTGTCATCGGAGGATATTG & (TTTGAA) 7 \\
\hline Ach64 & 39884 & AGCGAGTGACAGAACTATTT & AGAAGAGGTGTGGAGAAATT & (TTTTTG) 6 \\
\hline Ach65 & 6883 & TTTCATGACCCTCCCAATAG & CCAGGCATCCAGTACTTAAG & (AAAAATT) 6 \\
\hline Ach66 & 491 & CAGGAGTCAGCACAGAAATA & TTACCTGCCACTGCTATTAC & (ATTCATC) 5 \\
\hline Ach67 & 18925 & TGTCCTGGGGTAAGAAGATA & TGTGTTCCTCCTTCCAAAAT & (GAGTCTA) 5 \\
\hline Ach68 & 12652 & GGTTGAGTGGGTTGACTATT & AGAGCAAATCAAACCAACATTA & (TATCATC) 5 \\
\hline Ach69 & 18164 & ACTTGTTTCGAGCCTGATAA & TGAGTGACGTTGCAGAAATA & (TTTTTTC) 5 \\
\hline
\end{tabular}


Table 4. Locus diversity of 15 SSRs analyzed in three populations of Aristotelia chilensis.

\begin{tabular}{|c|c|c|c|c|c|c|c|c|c|}
\hline \multirow[b]{2}{*}{ Locus } & \multicolumn{3}{|c|}{$\begin{array}{l}\text { Pop. 1. Metropolitan Region } \\
\qquad(\mathrm{n}=20)\end{array}$} & \multicolumn{3}{|c|}{$\begin{array}{l}\text { Pop. 2. Maule Region } \\
\qquad(\mathrm{n}=20)\end{array}$} & \multicolumn{3}{|c|}{$\begin{array}{l}\text { Pop. 3. Los Lagos Region } \\
(\mathrm{n}=20)\end{array}$} \\
\hline & A & $\mathrm{He}$ & Но & A & $\mathrm{He}$ & Ho & A & $\mathrm{He}$ & Ho \\
\hline Ach3 & 6 & 0.725 & 0.389 & 7 & 0.758 & 0.700 & 4 & 0.675 & 0.650 \\
\hline Ach4 & 7 & 0.725 & 0.385 & 10 & 0.858 & 0.722 & 4 & 0.589 & 0.263 \\
\hline Ach5 & 5 & 0.681 & 0.714 & 8 & 0.799 & 0.529 & 3 & 0.526 & 0.611 \\
\hline Ach11 & 7 & 0.796 & 0.471 & 8 & 0.856 & 0.647 & 7 & 0.755 & 0.474 \\
\hline Ach14 & 5 & 0.691 & 0.583 & 7 & 0.831 & 0.733 & 7 & 0.741 & 0.421 \\
\hline $\operatorname{Ach} 28$ & 4 & 0.584 & 0.250 & 8 & 0.629 & 0.200 & 4 & 0.625 & 0.200 \\
\hline Ach29 & 4 & 0.722 & 0.421 & 4 & 0.600 & 0.611 & 2 & 0.499 & 0.350 \\
\hline Ach38 & 3 & 0.493 & 0.176 & 4 & 0.578 & 0.650 & 2 & 0.480 & 0.600 \\
\hline Ach 41 & 4 & 0.719 & 0.625 & 5 & 0.716 & 0.556 & 4 & 0.606 & 0.500 \\
\hline Ach45 & 5 & 0.520 & 0.450 & 4 & 0.694 & 0.700 & 1 & 0.000 & 0.000 \\
\hline Ach47 & 3 & 0.486 & 0.400 & 3 & 0.329 & 0.389 & 2 & 0.375 & 0.200 \\
\hline Ach54 & 3 & 0.486 & 0.300 & 2 & 0.188 & 0.211 & 1 & 0.000 & 0.000 \\
\hline Ach57 & 5 & 0.683 & 0.765 & 6 & 0.565 & 0.400 & 3 & 0.421 & 0.250 \\
\hline Ach61 & 5 & 0.719 & 0.684 & 3 & 0.204 & 0.222 & 3 & 0.405 & 0.500 \\
\hline Ach68 & 5 & 0.669 & 0.800 & 4 & 0.477 & 0.444 & 3 & 0.586 & 0.950 \\
\hline
\end{tabular}

A: Number of alleles; He: expected heterozygosity; Ho: observed heterozygosity; n: number of individuals considered in each population.

These microsatellite markers are not the first to be described in A. chilensis species, but the 11 available ones (Bastías et al., 2016) were unable to differentiate a group of seven A. chilensis accessions under domestication collected from different valleys in the South of Chile, which exhibited evident phenotypic differences; the new set of 15 SSRs presented here differentiated all but one pair of these domesticated accessions, partially fulfilling breeder's hypothesis, whom initially believed to have seven different accessions, turning out to be six -well defined- different genotypes (Figure 1).

The transferability of these markers in a wider genetic background was evaluated. For that purpose, representative samples of two related species belonging to Elaeocarpaceae family, Crinodendron patagua Molina and Aristotelia peduncularis (Labill.) Hook. f. were analyzed with the 15 polymorphic SSRs (Table 5). Crinodendron patagua is an endemic species of central Chile and results showed good amplification signal only in six out of 15 SSRs (Table 6).

Figure 1. Electrophoretic separation of alleles for seven Aristotelia chilensis domestication lines using two SSR markers. A) SSR marker Ach14; B) Ach28. Numbers and bars correspond to the different genotypes, with one to three replicates (different plants) each. Results revealed that samples \#5 and \#6 were non-differentiable based on 14 polymorphic markers, suggesting they are clones of the same material. Electrophoresis lasted $2 \mathrm{~h}$ at $75 \mathrm{~W}$ and $45 \mathrm{~mA} ; 3 \mu \mathrm{L}$ PCR product was loaded on each lane. DNA bands were silver-stained.

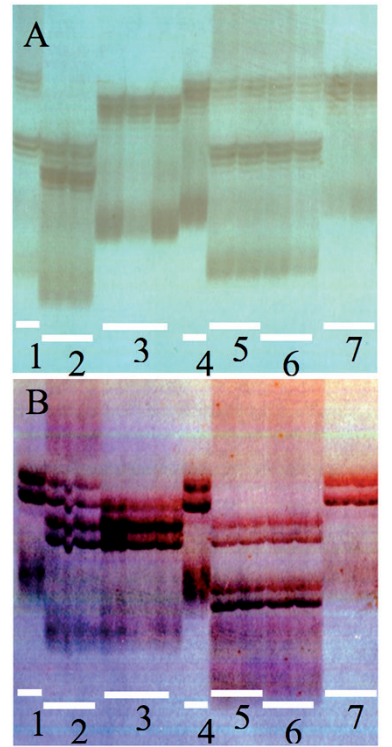


Table 5. Herbarium data for Aristotelia chilensis, A. peduncularis and Crinodendron patagua accessions.

\begin{tabular}{|c|c|c|c|}
\hline Species & Population code & Voucher nr & Geographical coordinates \\
\hline Aristotelia chilensis (Molina) Stuntz & Population 1 & $\begin{array}{l}\text { SGO } \\
169860\end{array}$ & $\begin{array}{l}33^{\circ} 29^{\prime} 45^{\prime \prime} \mathrm{S}, 70^{\circ} 28^{\prime} 57^{\prime \prime} \mathrm{W} \\
900 \mathrm{~m} \text { a.s.1 }\end{array}$ \\
\hline Aristotelia chilensis (Molina) Stuntz & Population 2 & $\begin{array}{l}\text { SGO } \\
169859\end{array}$ & $\begin{array}{l}34^{\circ} 50^{\prime} 18^{\prime \prime} \mathrm{S}, 71^{\circ} 21^{\prime} 17^{\prime \prime} \mathrm{W} \\
250 \text { m a.s. } 1\end{array}$ \\
\hline Aristotelia chilensis (Molina) Stuntz & Population 3 & $\begin{array}{l}\text { SGO } \\
169861\end{array}$ & $\begin{array}{l}41^{\circ} 40^{\prime} 08^{\prime \prime} \mathrm{S}, 73^{\circ} 03^{\prime} 50^{\prime \prime} \mathrm{W} \\
70 \mathrm{~m} \text { a.s. }\end{array}$ \\
\hline Aristotelia peduncularis (Labill.) Hook. f. & ND & $\begin{array}{l}\mathrm{HO} \\
569841\end{array}$ & $\begin{array}{l}42^{\circ} 55^{\prime} 20^{\prime \prime} \mathrm{S}, 147^{\circ} 15^{\prime} 11^{\prime \prime} \mathrm{W} \\
420 \mathrm{~m} \text { a.s.l }\end{array}$ \\
\hline Crinodendron patagua Molina & ND & $\begin{array}{l}\text { SGO } \\
169858\end{array}$ & $\begin{array}{l}33^{\circ} 24^{\prime} 27^{\prime \prime} \mathrm{S}, 70^{\circ} 36^{\prime} 20^{\prime \prime} \mathrm{W} \\
650 \mathrm{~m} \text { a.s. } 1\end{array}$ \\
\hline
\end{tabular}

Table 6. Transferability of Aristotelia chilensis SSR markers to related species, A. peduncularis and Crinodendron patagua (Elaeocarpaceae).

\begin{tabular}{|c|c|c|c|c|c|c|c|c|c|c|c|}
\hline \multirow[b]{3}{*}{ SSR markers } & \multicolumn{11}{|c|}{ PCR signal quality } \\
\hline & \multicolumn{5}{|c|}{ A. peduncularis } & \multicolumn{5}{|c|}{ C.patagua } & \multirow[t]{2}{*}{ A. chilensis } \\
\hline & 1 & 2 & 3 & 4 & 5 & 1 & 2 & 3 & 4 & 5 & \\
\hline Ach3 & $210-230$ & ++ & ++ & ++ & ++ & + & + & + & + & + & ++ \\
\hline Ach4 & $246-254$ & ++ & ++ & ++ & ++ & -- & -- & -- & -- & -- & ++ \\
\hline Ach5 & $246-260$ & ++ & ++ & ++ & ++ & $+/-$ & $+/-$ & $+/-$ & $+/-$ & $+/-$ & ++ \\
\hline Ach11 & $246-250$ & ++ & ++ & ++ & ++ & + & + & + & + & + & ++ \\
\hline Ach14 & + & + & + & + & + & $+/-$ & $+/-$ & $+/-$ & $+/-$ & $+/-$ & ++ \\
\hline Ach28 & $190-210$ & ++ & ++ & ++ & ++ & + & + & + & + & + & ++ \\
\hline Ach29 & $224-246$ & ++ & ++ & ++ & ++ & -- & -- & -- & -- & -- & ++ \\
\hline Ach38 & -- & -- & -- & -- & -- & -- & -- & -- & -- & $+/-$ & ++ \\
\hline Ach41 & $226-230$ & ++ & ++ & ++ & ++ & -- & -- & -- & -- & -- & ++ \\
\hline Ach45 & $160-180$ & ++ & ++ & ++ & ++ & -- & -- & -- & + & + & ++ \\
\hline Ach47 & $170-190$ & ++ & ++ & ++ & ++ & + & + & + & + & + & ++ \\
\hline Ach54 & -- & -- & -- & -- & -- & + & + & + & + & + & ++ \\
\hline Ach57 & $252-256$ & ++ & ++ & ++ & ++ & + & + & + & + & + & + \\
\hline Ach61 & $210-224$ & ++ & ++ & ++ & ++ & -- & -- & -- & -- & -- & ++ \\
\hline Ach68 & $196-206$ & ++ & ++ & ++ & ++ & -- & -- & -- & -- & $+/-$ & ++ \\
\hline
\end{tabular}

++: Clear and strong PCR signal; +: good signal; +/-: weak signal; --: no amplification.

The estimated allele sizes (bp) for each primers/species combination is indicated for sample \#1 of A.peduncularis.

Results of banding pattern showed an outstanding performance for A. peduncularis, native to Tasmania in Australia, with clear and strong PCR signals in most of the SSR analyzed (13 out of 15 SSRs amplified) (Table 6). These results are not unexpected, considering that the transferability of markers tend to be lower when phylogenetic distance is higher, such as relatives at the family level, compared to higher transferability usually observed in the framework of species of the same genus (in this case, Aristotelia sp.) This is commonly observed in many fruit crops and other taxonomical groups, a good example being the inter-specific hybrids used as rootstocks, as has been described in the genus Prunus spp. (Arismendi et al., 2012) and Vitis spp. (Lin and Walker, 1998; Gizella et al., 2011), among others. In these cases, a small set of SSR markers were able to tag the corresponding genomic regions and identify hybrids derived from different species.

\section{CONCLUSIONS}

A new set of 15 SSRs for Aristotelia chilensis species giving highly polymorphic allelic patterns in 60 individuals belonging to three populations from different geographical regions in Chile was obtained. It was possible to efficiently differentiate between many genotypes of maqui, including some specimens under domestication, making this set of markers the first useful tool for maqui genotypes traceability. Additionally, 13 out of 15 SSR markers showed clear and polymorphic amplification signals when analyzed in five specimens of the related species A. peduncularis, suggesting a high transferability of this new set of markers into new species of the same genus. 


\section{REFERENCES}

Arismendi, M.J., Hinrichsen, P., Almada, R., Pimentel, P., Pinto, M., and Sagredo, B. 2012. Characterization of genetic diversity of stone fruit rootstocks used in Chile by means of microsatellite markers. Journal of the American Society for Horticultural Sciences 137:302-310

Bastías, A., Correa, F., Rojas, P., Almada, R., Muñoz, C., and Sagredo, B. 2016. Identification and characterization of microsatellite loci in maqui (Aristotelia chilensis [Molina] Stuntz) using next-generation sequencing (NGS). PLOS ONE 11:e0159825.

Bastías, A., Correa, F., Rojas, P., Martin, C., Pérez-Diaz, J., Yáñez, C., et al. 2019. Draft genome sequence data of maqui (Aristotelia chilensis) and identification of SSR markers. Data in Brief 27:104545.https://doi.org/10.1016/j.dib.2019.104545.

Céspedes, C.L., El-Hafidi, M., Pavon, N., and Alarcon, J. 2008. Antioxidant and cardioprotective activities of phenolic extracts from fruits of Chilean blackberry Aristotelia chilensis (Elaeocarpaceae), Maqui. Food Chemistry 107:820-829.

Chikhi, R., and Medvedev, P. 2014. Informed and automated k-mer size selection for genome assembly. Bioinformatics 30(1):31.37. https://doi.org/10.1093/bioinformatics/btt310.

Fernández, M.P., Preller, C., Fischer, S., Espinoza, C., Pena-Rojas, K., and Menendez-Miguelez, M. 2019. Maqui (Aristotelia chilensis [Molina] Stuntz): the most antioxidant wild berry towards agricultural production. Fruits 74:214-226.

Fredes, C., Yousef, G.G., Robert, P., Grace, M.H., Lila, M.A., Gómez, M., et al. 2014. Anthocyanin profiling of wild maqui berries (Aristotelia chilensis [Mol.] Stuntz) from different geographical regions in Chile. Journal of the Science of Food and Agriculture 94:2639-2648.

Gizella, J., Molnár, K.G., Májer, J., Szoke, B., Tarczal, E., Varga, P., et al. 2011. Analysis of grape rootstocks by SSR markers. Journal International des Science de la Vigne et duVin 45:1-12.

Gugerli, F., Rüegg, M., and Vendramin, G.G. 2009. Gradual decline in genetic diversity in Swiss stone pine populations (Pinus cembra) across Switzerland suggests postglacial re-colonization into the Alps from a common eastern glacial refugium. Botanica Helvetica 119:13.

Hewitt, G.M. 2004. Genetic consequences of climatic oscillations in the Quaternary. Philosophical Transactions of the Royal Society of London. Series B, Biological Sciences 359:183-195. doi:10.1098/rstb.2003.1388.

Lin, H., and Walker, M.A. 1998. Identifying grape rootstocks with simple sequence repeat (SSR) DNA markers. American Journal of Enology and Viticulture 49:403-407.

Lodhi, M.A., Ye, G.N., Weeden, N.F., and Reisch, B.I. 1994. A simple and efficient method for DNA extraction from grapevine cultivars and Vitis species. Plant Molecular Biology Reporter 12:6-13.

Miranda-Rottmann, S., Aspillaga, A.A., Pérez, D.D., Vasquez, L., Martinez, A.L., and Leighton, F. 2002. Juice and phenolic fractions of the berry Aristotelia chilensis inhibit LDL oxidation in vitro and protect human endothelial cells against oxidative stress. Journal of Agricultural and Food Chemistry 50:7542-7547.

Misle, E., Garrido, E., Contardo, H., and González, W. 2011. Maqui (Aristotelia chilensis (Mol.) Stuntz) the amazing Chilean tree: a review. Journal of Agricultural Science and Technology B 1:473-482.

Moreno, P.I., Videla, J., Valero-Garcés, B., Alloway, B.V., and Heusser, L.E. 2018. A continuous record of vegetation, fire-regime and climatic changes in northwestern Patagonia spanning the last 25,000 years. Quaternary Science Reviews 198:15-36.

Peakall, R., and Smouse, P.E. 2012. GenAlEx 6.5: A genetic analysis in Excel. Population genetic software for teaching and research-an update. Bioinformatics 28:2537-2539.

Rojo, L.E., Ribnicky, D., Logendra, S., Poulev, A., Rojas-Silva, P., Kuhn, P., et al. 2012. In vitro and in vivo anti-diabetic effects of anthocyanins from Maqui Berry (Aristotelia chilensis). Food Chemistry 131:387-396.

Rozen, S., and Skaletsky, H. 2000. Primer3 on the WWW for general users and for biologist programmers. Bioinformatics Methods and Protocols 132:365-386.

Salgado, P., Prinz, K., Finkeldey, R., Ramírez, C.C., and Vogel, H. 2017. Genetic variability of Aristotelia chilensis ("maqui”) based on AFLP and chloroplast microsatellite markers. Genetic Resources and Crop Evolution 64:2083-2091.

Thiel, T., Michalek, W., Varshney, R., and Graner, A. 2003. Exploiting EST databases for the development and characterization of gene-derived SSR-markers in barley (Hordeum vulgare L.) Theoretical and Applied Genetics 106:411-422.

Tóth, E.G., Tremblay, F., Housset, J.M., Bergeron, Y., and Carcaillet, C. 2019. Geographic isolation and climatic variability contribute to genetic differentiation in fragmented populations of the long-lived subalpine conifer Pinus cembra L. in the western Alps. BMC Evolutionary Biology 19:190. doi:10.1186/s12862-019-1510-4.

Vogel, H., González, B., Catenacci, G., and Doll, U. 2016. Domestication and sustainable production of wild crafted plants with special reference to the Chilean maqui berry (Aristotelia chilensis). Julius-Kühn-Archiv 50:50-52.

Zimin, A.V., Marçais, G., Puiu, D., Roberts, M., Salzberg, S.L., and Yorke, J.A. 2013. The MaSuRCA genome assembler. Bioinformatics 29(21):2669-2677. https://doi.org/10.1093/bioinformatics/btt476. 